\title{
Frailty and Spousal/Partner Bereavement in Older People: A Systematic Scoping Review Protocol
}

\author{
Vseteckova J*, Mahon A, Boyle G, Jones K and Garcia R
}

Senior Lecturer Health, Wellbeing and Social Care, Faculty of Wellbeing, Education and Language Studies, The Open University, UK

*Corresponding author: Vseteckova J, Senior Lecturer Health, Wellbeing and Social Care, Faculty of Wellbeing, Education and

Language Studies, The Open University, Room 024, Horlock Building, Walton Hall, Milton Keynes, MK7 6AA, UK

\begin{abstract}
ARTICLE INFO
Received: 㗀 January 04, 2020

Published: 幽 January 13, 2020

Citation: Vseteckova J, Mahon A, Boyle G, Jones K, Garcia R. Frailty and Spousal/ Partner Bereavement in Older People: A

\section{ABSTRACT}

\section{Aims of the Proposed Review}

The aim of this project is to conduct a systematic review that will identify and collate published information relating to frailty and spousal/partner bereavement (i.e. the death of a life partner whether married or unmarried co-habiting) in older people (aged 60 years and above). The review will include all relevant national (UK) and international research. This review aims to identify any gaps in literature that can help inform future healthcare policies and models.
\end{abstract} Systematic Scoping Review Protocol. Biomed J Sci \& Tech Res 24(4)-2020. BJSTR. MS.ID.004075.
Keywords: Frailty Research; Spectrum Syndrome; Psychological Distress; Frailty Index

\section{Introduction}

According to the World Health Organisation, 900 million people globally were aged 60 years or more in 2015 [1]. This is predicted to reach 2 billion by 2050 [2]. The population of the United Kingdom is getting older. It is projected that by 2036, over half of all local authorities in the UK will have $25 \%$ or more of their local population aged 65 and over (Office for National Statistics, 2017). The increasing number of international ageing populations has resulted in a growing interest in frailty research. The syndrome known as 'frailty' does not have an agreed operational definition nor agreed diagnostic criteria [3-5]. Frailty is an ambiguous term however; it generally refers to an increased vulnerability to adverse health outcomes and is most commonly identified in older adults. Frailty is not determined by old age. Frailty is a spectrum syndrome that can encompass a myriad of environmental, psychological and physiological diagnosis. While it is estimated that up to three quarters of people over 85 years might not be frail, individuals who are frail have significantly increased risks of falls, disability, longterm care and death [6-7]. How frailty develops and how it can be prevented remains unclear.
Research examining the specific relationship between marital status and frailty appears to be limited [8]. Trevisan and colleagues found a gender-specific difference in the onset of frailty, which were also noted in marital-status and mortality and psychological wellbeing studies, reporting increased risk for divorced, single, widowed or never married males compared to females $[9,10]$. While frailty appears to universally affect females more than males, Trevisan and colleagues found that widowed or single males have a higher risk of developing frailty compared to married males while widowed women carry a significantly lower risk of becoming frail compared to married women. There is extensive research demonstrating a negative impact of widowhood on health outcomes including higher risk of disability [11] and higher rates of depression and psychological distress [10,12] and mortality in separated individuals compared to married individuals [10]. Following the PICO framework [13] and PRISMA-P methodology [14], this systematic review aims to synthesise existing knowledge, identify gaps in the literature and provide recommendations for future research relating to frailty and spousal/partner bereavement 
(i.e. the death of a life partner whether married or unmarried cohabiting) in older people (aged 60 years and above).

\section{Methods}

Employing a systematic review methodology, we aim to identify and collate published information relating to frailty and spousal/ partner bereavement (i.e. the death of a life partner whether married or unmarried co-habiting) in older people (aged 60 years and above). While preliminary searches suggested that research on this topic is limited it appears to indicate a negative association between marital-status (i.e. widowhood) and frailty. The review followed the Preferred Reporting Items for Systematic Reviews and Meta-analysis for Protocols (PRISMA-P) guidelines with the PRISMA-P checklist being used to draft the current protocol [14]. The PICO framework was used to establish the questions the review will address. The review aims to collate all available information. Therefore, all research methodologies, including possible intervention studies, will be included i.e. RCTs, quantitative studies, observational studies, qualitative studies, mixed-method studies and any other form of study that specifically examines the relationship between frailty and marital status. Interventions may include medical/physical, social, psychological or a combination of all three. While a systematic review methodology was used, the review was broad in its scope. For this reason, the protocol and review design included the methodological framework proposed by Arksey and O'Malley [5]. The Arksey and O'Malley's framework consists of five stages:

1. identifying the research question,

2. identifying relevant studies,

3. study selection,

4. charting the data,

5. collating, summarising and reporting results.

Each stage is discussed in further detail below. The last optional stage, consultation, was not included in the current review.

\section{Identifying the Research Question}

The aim of the current review is to collect and synthesise current knowledge on frailty and spousal/partner bereavement (i.e. the death of a life partner whether married or unmarried cohabiting) in older people (aged 60 years and above). This includes determining the outcomes, if any, of spousal/partner bereavement on frailty, identifying the factors that protect against and/or increase frailty in older bereaved populations and identifying the barriers and facilitators that can influence access to interventions to reduce, slow-down or reverse frailty in these bereaved populations (if any interventions exist and if a relationship between frailty and spousal/partner bereavement is identified). The review aims to synthetize existing knowledge, identify gaps in the literature and provide recommendations for future research, which may lead to improved interventions.
To meet these objectives, this review asked the following questions:

1) Is there a relationship between spousal/partner bereavement and frailty?

2) What factors influence frailty in bereaved older adults? (protective or other)

3) What interventions are available within the UK and internationally, that prevent any impact of spousal/partner bereavement and frailty?

\section{Identifying Relevant Studies}

The review included a search of electronic databases (see section 3.2.4), reference lists (ancestor searching), website organisations and conference proceedings. Articles and evaluation reports related to the topic of spousal/partner bereavement and frailty were identified through an initial exploratory online search using the electronic databases MEDLINE (PubMed) and CINAHL. The text words in the title and abstract of relevant retrieved papers were then analysed as well as the index terms used to describe the articles. All identified keywords and index terms were used to develop a rigorous search strategy that was undertaken across all included databases. The reference list of identified reports and articles was also searched for additional studies. The search was limited to literature written in English. The search strategy can be found in Appendix.

\section{Inclusion Criteria}

\section{Types of Participants}

Eligible participants included:

1) Older adults (60 years and above)

2) Marital status as married, civil partnership, widow, widower, widowed or single if referring to the bereavement of a co-habiting spouse/partner (unmarried un-cohabiting)

3) Participants must have co-habited with their spouse prior to bereavement (with the exception of short-term hospitalisations prior to death)

\section{Concept}

A. Types of Outcome Measures

The primary outcome of interest was frailty. All frailty definitions and assessment tools are included in addition to studies that included subjective definitions of frailty or studies that do not detail the frailty criteria used. The two most frequently used frailty definitions and assessment tools are the frailty phenotype (also known as Fried's definition or Cardiovascular Health Study (CHS) definition [6] and the frailty [16] The frailty phenotype classifies frailty as a syndrome that has three or more of five phenotypic criteria: weakness as measured by low grip strength, slowness by slowed walking speed, low level of physical activity, low energy or 
self-reported exhaustion, and unintentional weight loss. Pre-frailty is defined as having one or two criteria present. Non-frail older adults are classified as having none of the above five criteria.

The frailty index is a measure of the number of deficits identified during a comprehensive geriatric assessment, including diseases, physical and cognitive impairments, psychosocial risk factors, and common geriatric syndromes other than frailty $[16,17]$. Variables are identified as meeting the FI inclusion deficit criteria if the variable needs to be acquired, is age-associated, is associated with an adverse outcome, and should not saturate too early [17-19].

Context/Setting: The review is international in scope.

Types of studies: To provide a comprehensive overview of this research topic all existing literature will be included, e.g. primary research studies, systematic reviews, meta-analyses, letters, guidelines, websites etc.

\section{Electronic Searches}

The following electronic databases will be searched:
a) CINAHL
b) British Nursing Index
c) Web of Knowledge
d) Cochrane library
e) PsychInfo
f) SocIndex
g) University of York Centre for Reviews and Dissemination (DARE, NHS EED, HTA)

h) JBI Database of Systematic Reviews and Implementation Reports,
i) MEDLINE
j) EPPI
k) Epistemonikos

\section{Searching other resources}

Grey literature will be searched in OpenGrey, Google, and Google Scholar. A full citation and reference search will be conducted for any papers included in the final review.

\section{Stage 3: Study Selection}

Study Screening and Selection: Initial screening selection (title and abstract screening) will be distributed amongst four reviewers divided into two groups. Each group will screen the full initial screening selection, with hits divided amongst both reviewers in each group. The screening selection for reviewer one from group A will be paired with reviewer one from Group $\mathrm{B}$ and similarly for reviewer two from group A and reviewer two from Group B. This allows a measure of inter-rater relability, using
Cohen's kappa coefficient ( $\kappa$ ), for both sets of paired reviewers. After eliminating the duplicates (studies identified more than once by the search engines), an initial screening of titles, abstracts, and summaries (if applicable) will be undertaken to exclude records that clearly do not meet the inclusion criteria. Each record will be classified as 'include' or 'exclude' to identify relevant and exclude irrelevant literature. The researchers will be inclusive at this stage and, if uncertain about the relevance of a publication or report, it will be left in. Any disagreements in studies shortlisted for full text screening will be solved by consensus or by the decision of a fifth reviewer where necessary.

The full text will be obtained for all the records that potentially meet the inclusion criteria (based on the title and abstract/ summary only), as agreed by all reviewers. In this second step, all the full text papers will be screened against the inclusion criteria, using a standardised tool. Studies that do not meet the inclusion criteria will be listed with the reasons for exclusion. Multiple publications and reports on the same interventions will be linked together and compared for completeness. The record containing the most complete data on any single intervention will be identified as the primary article in the review, which can usually be the original study or most recent evaluation report. A PRISMA-P flow-chart of study selection will be included in the review.

\section{Stage 4: Charting the Data}

Data Extraction and Management: Data for analysis will be extracted from the included studies and managed in an Excel spread sheet. A data extraction sheet will be developed, tailored to the requirements of the review. The data extraction sheet will be tested on three included papers and, where necessary, it will be revised to ensure it can be reliably interpreted and could capture all relevant data from different study designs. Extracted data will include authors, year of study/report, aim/purpose, type of paper (e.g. journal article, annual evaluation report, etc), country/location, study population (e.g., age of participants, gender, marital status, living arrangements, health status pre-bereavement), average length of relationship (in years), average length of bereavement (in years) sample size, study design, frailty definition/criteria, frailty rate, factors that impact on frailty rate (protective and negative factors), description of any interventions/services/support for study population, description of the interventions/services/ support (if any), factors that facilitate and/or hinder access to interventions/services/support (if any), key findings that relate to the review questions.

\section{Stage 5: Collating, Summarising and Reporting the Results}

Presentation of the Results (Data Synthesis): Findings from included studies will be synthesised narratively. First, a preliminary synthesis will be conducted to develop an initial description of the findings of included records and to organise them so that patterns across records could be identified. In a second step, thematic 
analysis will be used to analyse the findings. The following five steps of thematic analysis will be followed adopting a recursive process [17]:

1. Familiarisation with the extracted data

2. Generation of initial codes

3. Searching for themes

4. Reviewing themes

5. Defining and naming themes.

Following Arksey and O'Malleys (2005) suggested framework, a template (using Microsoft Excel) will be used to gather the themed findings, and the final outcomes from the systematic scoping review will be published in the academic literature. Depending on the findings available the reviewers will aim to provide a flow chart mapping the available information relating to frailty and spousal/ partner bereavement including the needs identified.

\section{References}

1. (2018) Ageing and health. World Health Organization.

2. (2017) Global strategy and action plan on ageing and health. World Health Organization p. 1-46.

3. Bergman H, Ferrucci L, Guralnik J, Hogan DB, Hummel S, et al. (2007) Frailty: an emerging research and clinical paradigm-issues and controversies. The Journals of Gerontology Series A: Biological Sciences and Medical Sciences 62(7): 731-737.

4. Buckinx F, Rolland Y, Reginster JY, Ricour C, Petermans J, et al. (2015) Burden of frailty in the elderly population: perspectives for a public health challenge. Archives of Public Health 73(1): 19.

5. Hogan DB, MacKnight C, Bergman H (2003) Models, definitions, and criteria of frailty. Aging Clin Exp Res 15(3 suppl): 1-29.

6. Fried LP, Tangen CM, Walston J, Newman AB, Hirsch C, et al. (2001) Frailty in older adults: evidence for a phenotype. J Gerontol A Biol Sci Med Sci 56(3): 146-156.

\section{ISSN: 2574-1241}

DOI: $10.26717 /$ BJSTR.2020.24.004075

Vseteckova J. Biomed J Sci \& Tech Res

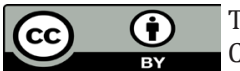

This work is licensed under Creative Commons Attribution 4.0 License

Submission Link: https://biomedres.us/submit-manuscript.php
7. Song X, Mitnitski A, Rockwood K (2011) Nontraditional risk factors combine to predict Alzheimer disease and dementia. Neurology 77(3): 227-234.

8. Trevisan C, Veronese N, Maggi S, Baggio G, De Rui M, et al. (2016) Marital status and frailty in older people: gender differences in the Progetto Veneto Anziani Longitudinal Study. Journal of Women's Health 25(6): 630-637.

9. Hu YR, Goldman N (1990) Mortality differentials by marital status: An international comparison. Demography 27(2): 233-250.

10. Gove WR (1973) Sex, marital status, and mortality. AJS 79(1): 45-67.

11. Goldman N, Korenman S, Weinstein R (1995) Marital status and health among the elderly. Soc Sci Med 40(12): 1717-1730.

12. Pearlin LI, Johnson JS (1977) Marital status, life-strains and depression. Am Sociol Rev 42(5): 704-715.

13. Richardson WS, Wilson MC, Nishikawa J, Hayward RS (1995) The wellbuilt clinical question: a key to evidence-based decisions. ACP journal club 123(3): A12-A13.

14. Shamseer L, Moher D, Clarke M, Ghersi D, Liberati A, et al. (2015) Preferred reporting items for systematic review and meta-analysis protocols (PRISMA-P) 2015: elaboration and explanation. Bmj 349: 7647.

15. Arksey H, O Malley L (2005) Scoping studies: towards a methodological framework. Int J Soc Res Methodol 8(1): 19-32.

16. Jones DM, Song X, Rockwood K (2004) Operationalizing a frailty index from a standardized comprehensive geriatric assessment. J Am Geriatr Soc 52(11): 1929-1933.

17. Searle SD, Mitnitski A, Gahbauer EA, Gill TM, Rockwood K (2008) A standard procedure for creating a frailty index. BMC Geriatr 8(24).

18. Chen X, Mao G, Leng SX (2014) Frailty syndrome: an overview. Clinical interventions in aging $9(9)$ : 433-441.

19. Shor E, Roelfs DJ, Curreli M, Clemow L, Burg MM, et al. (2012) Widowhood and mortality: a meta-analysis and meta-regression. Demography 49(2): 575-606.

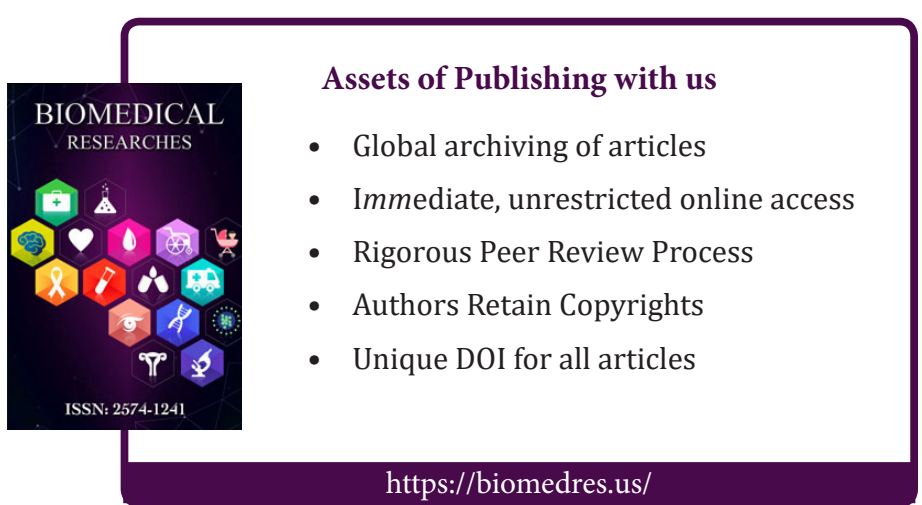

